LPTENS 93/14

ROME prep. 93/946

LPTHE Orsay- $93 / 20$

SHEP 92/93-25

WUB 93-27 July 1993.

\title{
Semi-leptonic Decays of Heavy Flavours on a Fine Grained Lattice
}

\author{
As.Abada $^{1}$, C.R.Allton ${ }^{2}$, Ph.Boucaud ${ }^{1}$, D.B.Carpenter ${ }^{3}$, \\ M.Crisafulli ${ }^{2}$, S.Güsken ${ }^{4}$, P.Hernandez ${ }^{5}$, V.Lubicz ${ }^{2}$, G.Martinelli ${ }^{2,6}$, \\ O.Pène $^{1}$, C.T.Sachrajda ${ }^{7}$, K.Schilling ${ }^{4}$, G.Siegert ${ }^{4}$, R.Sommer ${ }^{8}$. \\ ${ }^{1}$ LPTHE, Orsay, France,凹 \\ 2 Dip. di Fisica, Univ. di Roma 'La Sapienza', \\ I-00185 Roma,Italy and INFN, Sezione di Roma, Italy. \\ ${ }^{3}$ Dept. of Electronics and Computer Science, \\ The University, Southampton SO9 5NH, UK. \\ ${ }^{4}$ Physics Dept., Univ. of Wuppertal, D-42097 Wuppertal, Germany. \\ ${ }^{5}$ Dept. de Fís. Teórica C-XI, Univ. Autónoma de Madrid, E-28049, Madrid ,Spain. \\ ${ }^{6}$ Laboratoire de Physique Théorique de l'École Normale Supérieure?. \\ 24 rue Lhomond, 75231 Paris CEDEX 05, France. \\ 7 Dept. of Physics, The University, Southampton SO9 5NH, UK. \\ 8 Deutsches Elektronen-Synchrotron, DESY, Notkestrasse 85,D-22603 Hamburg, Germany.
}

\begin{abstract}
We present the results of a numerical calculation of semi-leptonic form factors relevant for heavy flavour meson decays into light mesons. The results have been obtained by studying two- and three-point correlation functions at $\beta=6.4$ on a $24^{3} \times 60$ lattice, using the Wilson action in the quenched approximation.

From the study of the matrix element $\left\langle K^{-}\left|J_{\mu}\right| D^{0}>\right.$ we obtain $f_{K}^{+}(0)=$ $0.65 \pm 0.18$. From the matrix element $\left\langle\bar{K}^{* 0}\left|J_{\mu}\right| D^{+}>\right.$we obtain $V(0)=$ $0.95 \pm 0.34, A_{1}(0)=0.63 \pm 0.14$ and $A_{2}(0)=0.45 \pm 0.33$. We also obtain $A_{1}\left(q_{\max }^{2}\right)=0.62 \pm 0.09, V(0) / A_{1}(0)=1.5 \pm 0.28$ and $A_{2}(0) / A_{1}(0)=$ $0.7 \pm 0.4$. The results for $f_{K}^{+}(0), V(0)$ and $A_{1}(0)$ are consistent with the experimental data and with previous lattice determinations with larger lattice spacings. In the case of $A_{2}(0)$ the errors are too large to draw any
\end{abstract}

\footnotetext{
${ }^{1}$ Laboratoire associé au Centre National de la Recherche Scientifique.

${ }^{2}$ Unité Propre du Centre National de la Recherche Scientifique, associée à l'École Normale Supérieure et à l'Université de Paris-Sud.
} 
firm conclusion. We also show, with the help of the heavy quark effective theory (HQET), that it is possible to extrapolate the form factors to the B meson. Our calculations show that the form factors follow a behaviour compatible with the predictions from HQET. Within large uncertainties, our results suggest that $A_{2} / A_{1}$ increases with the heavy quark mass. For $\mathrm{B}$ mesons $A_{2} / A_{1}$ can be as large as 1.5-2.0. We also get very rough estimates for the partial decay widths $\Gamma\left(B \rightarrow \pi l \nu_{l}\right)=\left|V_{u b}\right|^{2}(12 \pm 8) \times 10^{12} s^{-1}$ and $\Gamma\left(B \rightarrow \rho l \nu_{l}\right)=\left|V_{u b}\right|^{2}(13 \pm 12) \times 10^{12} s^{-1}$, which can be used to give upper bounds on the rates. 


\section{Introduction}

Semi-leptonic decays of heavy-light mesons have attracted considerable interest in the past years as they play a crucial role in the determination of the CabibboKobayashi-Maskawa mixing matrix and in the understanding of weak decays. Moreover, the study of the dependence of the form factors on the heavy quark mass checks the validity of the scaling laws, predicted by the heavy quark effective theory, in the range of masses corresponding to D and B mesons.

There is increasing evidence, that lattice QCD allows ab initio quantitative predictions of weak decay matrix elements [1]. Exclusive semi-leptonic decay channels of heavy flavour mesons have been studied in a series of papers [2]- [6], at a lattice resolution of $a^{-1} \sim 2-3 \mathrm{GeV}$. In order to improve the control over discretization errors, we present here a study on pseudoscalar-pseudoscalar as well as pseudoscalar-vector weak form factors, on a lattice $24^{3} \times 60$, at $\beta=6.4$, which corresponds to $a^{-1} \simeq 3.6-3.7 \mathrm{GeV}$. This is a continuation of our recent work [B] on the leptonic decay constants.

The main results of the present study are given in the abstract and in tables 9 , 10 and 12. These results have been obtained by extrapolating the matrix elements in momentum transfer, in the light and heavy quark masses. We have included in tables 18 all the numbers obtained from the direct study of the two- and threepoint correlation functions, computed at several values of $K_{W}$. These tables may be useful to check our results. We also give the details of the extrapolation, in order to allow the reader to reproduce the form factors in table 9 from the meson masses and the form factors of tables 1 . 8 . In section 3.2 we show that it is possible to extrapolate the form factors to the B meson using the Heavy Quark Effective Theory (HQET). In this way one can get also informations on the corrections to the infinite mass limit. Due to large statistical errors, we are only able to give a rough estimate for the branching ratios of the processes $B \rightarrow \pi$ and $B \rightarrow \rho$.

\section{Description of the Calculation}

\subsection{Lattice Setup}

We work with the standard Wilson action at $\beta=6.4$ for the gauge fields and the quark propagators 9, in the quenched approximation. We have generated 15 independent gauge field configurations on a $24^{3} \times 30$ lattice, separated by 5001600 sweeps, using the overrelaxed algorithm[10]. The 15 configurations were produced in groups of 5 , with three independent initial conditions. In each of the three cases the first of the useful configurations was obtained after an initial thermalization of at least 3000 sweeps ( 500 with the Metropolis algorithm and 2500 with the overrelaxed algorithm ).

On each configuration we have computed the quark propagators for 7 different 
values of the Wilson hopping parameter $K_{W}$, corresponding to "heavy" quarks, $K_{H}=0.1275,0.1325,0.1375,0.1425$, and "light" quarks, $K_{L}=0.1485,0.1490$ and 0.1495 . Periodic boundary conditions on a $24^{3} \times 60$ lattice have been imposed in the calculation of the quark propagators, by using appropriate combinations of periodic and antiperiodic (in the time direction) quark propagators calculated on a $24^{3} \times 30$ lattice [1]. Further details on the lattice calibration, fitting procedures, mass spectrum, extraction of matrix elements of local operators between the vacuum and meson states, e.g. $\left\langle M_{P}\left|\bar{Q} \gamma_{5} q\right| 0>\right.$, can be found in ref. $[8]$.

\subsection{Form Factor Evaluation}

From the study of three-point correlation functions [2]-[6], one extracts the weak current matrix elements for a given momentum transfer:

$$
\begin{aligned}
<K\left|J_{\mu}\right| D>= & \left(p_{D}+p_{K}-\frac{M_{D}^{2}-M_{K}^{2}}{q^{2}} q\right)_{\mu} f_{K}^{+}\left(q^{2}\right)+\frac{M_{D}^{2}-M_{K}^{2}}{q^{2}} q_{\mu} f_{K}^{0}\left(q^{2}\right) \\
<K_{r}^{*}\left|J_{\mu}\right| D> & =e_{r}^{\beta}\left[\frac{2 V\left(q^{2}\right)}{M_{D}+M_{K^{*}}} \epsilon_{\mu \gamma \delta \beta} p_{D}^{\gamma} p_{K^{*}}^{\delta}+i\left(M_{D}+M_{K^{*}}\right) A_{1}\left(q^{2}\right) g_{\mu \beta}\right. \\
& \left.-i \frac{A_{2}\left(q^{2}\right)}{M_{D}+M_{K^{*}}} P_{\mu} q_{\beta}+i \frac{A\left(q^{2}\right)}{q^{2}} 2 M_{K^{*}} q_{\mu} P_{\beta}\right],
\end{aligned}
$$

where $q$ is the momentum transfer, $q=p_{D}-p_{K}$ or $q=p_{D}-p_{K^{*}}, P=p_{D}+p_{K^{*}}$ and $e_{r}^{\beta}$ is the polarization vector of the $K^{*} \cdot f_{K}^{+, 0}, V, A_{1,2}$ and $A$ are dimensionless form factors in the helicity basis, see for example [3]. From the matrix elements (11) and (2), by varying the Lorentz component of the current, the meson momenta and the $K^{*}$ polarization, one can extract the form factors.

In the following we give those details of the calculation which are specific to semi-leptonic decays and cannot be found in ref. [8].

The matrix elements have been computed for a D meson at rest using a pseudoscalar density as source, at a time distance $\left(t_{D}-t_{K, K^{*}}\right) / a=28$. The position of the light meson source is fixed in the origin and we have varied the time position (in the interval $t_{J} / a=12-16$ ) and the momentum of the weak current. For the $K$-source we have also used the pseudoscalar density and for the $K^{*}$ the local vector current. For the weak axial current we have chosen the local operator $Z_{A} \bar{Q}(x) \gamma_{\mu} \gamma_{5} q(x)$, while for the weak vector current we have used both the local $\left(Z_{V} \bar{Q}(x) \gamma_{\mu} q(x)\right)$ and the "conserved " currents. $Z_{A}$ and $Z_{V}$ are the renormalization constants of the axial and vector current respectively 12 , 13. The values quoted for the form factors are based on the choice $Z_{A}=0.88$ and $Z_{V}=0.84[14]$ - [16] which will be justified below.

The two- and three-point correlation functions have been computed for different spatial momenta which are allowed by the lattice discretization and volume.

\footnotetext{
${ }^{3}$ The latter would be conserved on the lattice in the limit of degenerate quark masses [12, 13].
} 


\begin{tabular}{|c|c|c|c|c|}
\hline$K_{1}=K_{2}$ & momentum & $E_{5}$ & $\bar{E}_{5}$ & $Z_{5} \times 10^{3}$ \\
\hline \multirow{3}{*}{0.1485} & $0,0,0$ & $0.27(1)$ & $0.27(1)$ & $3.9(0.3)$ \\
& $1,0,0$ & $0.39(2)$ & $0.38(1)$ & $5.7(1.6)$ \\
& $1,1,0$ & $0.41(4)$ & $0.46(1)$ & $2.5(1.6)$ \\
\hline \multirow{3}{*}{0.1490} & $0,0,0$ & $0.23(1)$ & $0.23(1)$ & $3.3(0.3)$ \\
& $1,0,0$ & $0.37(3)$ & $0.35(1)$ & $5.4(2.5)$ \\
& $1,1,0$ & $0.37(5)$ & $0.44(1)$ & $1.7(1.3)$ \\
\hline \multirow{3}{*}{0.1495} & $0,0,0$ & $0.19(1)$ & $0.19(1)$ & $2.9(0.4)$ \\
& $1,0,0$ & $0.36(5)$ & $0.33(1)$ & $5.3(5.2)$ \\
& $1,1,0$ & $0.32(5)$ & $0.42(1)$ & $1.1(0.9)$ \\
\hline
\end{tabular}

Table 1: Pseudoscalar meson masses and energies in lattice units and $Z_{5}$ from a fit to two-point correlations at different momenta and quark masses. The mesons are composed of two degenerate light quarks, and the Wilson parameter is given in the first column.

By defining $\vec{p}_{K, K^{*}}=2 \pi /(L a) \times\left(n_{x}, n_{y}, n_{z}\right)$, with $L=24$, we have used for $\vec{p}_{K, K^{*}}$ the following assignments $(0,0,0),(1,0,0),(0,1,0),(0,0,1),(1,1,0),(1,0,1), \ldots$, $(2,0,0), \ldots$, and $(1,1,1)$. We have combined different correlation functions which can be related by the spatial orthogonal group and parity. In all we have five independent momenta which in the following we will denote by $(0,0,0),(1,0,0)$, $(2,0,0),(1,1,0)$ and $(1,1,1)$.

Large momenta in lattice units imply large systematic errors. In order to monitor lattice artefacts at large momenta, we have fitted the light pseudoscalar meson two-point functions to the asymptotic form (at large time distances):

$$
\begin{aligned}
G_{5}(t, \vec{p}) & =\sum_{\vec{x}} e^{i \vec{p} \cdot \vec{x}}<P_{5}(\vec{x}, t) P_{5}^{\dagger}(\overrightarrow{0}, 0)> \\
& \rightarrow Z_{5} \frac{e^{-E_{5} T / 2}}{E_{5}} \cosh \left(E_{5}(t-T / 2)\right),
\end{aligned}
$$

where $P_{5}=\bar{q}(x) \gamma_{5} q^{\prime}(x), T$ is the time lattice length and $E_{5}$ the meson energy. The corresponding formula for the vector meson can be easily derived:

$$
\begin{array}{r}
G_{i j}(t, \vec{p})=\sum_{\vec{x}} e^{i \vec{p} \cdot \vec{x}}<V_{i}(\vec{x}, t) V_{j}^{\dagger}(\overrightarrow{0}, 0)>\rightarrow \\
\left(-g_{i j}+\frac{p_{i} p_{j}}{M_{V}^{2}}\right) Z_{V V} \frac{e^{-E_{V} T / 2}}{E_{V}} \cosh \left(E_{V}(t-T / 2)\right),
\end{array}
$$

where $V_{i}=\bar{q}(x) \gamma_{i} q^{\prime}(x)$.

An estimate of the lattice effects is obtained from a comparison of $E_{5}$, obtained from eq.(3i), with $\bar{E}_{5}=\sqrt{M_{5}^{2}+\vec{p}^{2}}$, where $M_{5}$ is the value of $E_{5}$ found from 


\begin{tabular}{|c|c|c|c|c|}
\hline$K_{1}=K_{2}$ & momentum & $E_{V}$ & $\bar{E}_{V}$ & $Z_{V V} \times 10^{3}$ \\
\hline \multirow{3}{*}{0.1485} & $0,0,0$ & $0.33(1)$ & $0.33(1)$ & $1.5(2)$ \\
& $1,0,0$ & $0.42(1)$ & $0.42(1)$ & $1.6(2)$ \\
& $1,1,0$ & $0.51(1)$ & $0.49(1)$ & $2.1(5)$ \\
\hline \multirow{3}{*}{0.1490} & $0,0,0$ & $0.30(1)$ & $0.30(1)$ & $1.2(1)$ \\
& $1,0,0$ & $0.40(1)$ & $0.40(1)$ & $1.3(2)$ \\
& $1,1,0$ & $0.50(2)$ & $0.48(1)$ & $2.0(6)$ \\
\hline \multirow{3}{*}{0.1495} & $0,0,0$ & $0.27(1)$ & $0.27(1)$ & $0.9(1)$ \\
& $1,0,0$ & $0.38(1)$ & $0.38(1)$ & $1.1(2)$ \\
& $1,1,0$ & $0.51(2)$ & $0.46(1)$ & $2.3(8)$ \\
\hline
\end{tabular}

Table 2: Vector meson masses and energies in lattice units and $Z_{V V}$ from a fit to two-point correlations at different momenta and quark masses. The mesons are composed of two degenerate light quarks, and the Wilson parameter is given in the first column.

a fit at $\vec{p}=0$. Moreover the values of $Z_{5}$ obtained by fitting the two-point functions at different momenta should be consistent (similarly for vector mesons). In analyzing the two-point correlation functions, we found that for $(1,1,1)$ and $(2,0,0)$ the results are too noisy with the present statistics and therefore they have not been used. In tables 1 and 2 we report, for different light quark masses, $E_{5}\left(E_{V}\right)$ and $Z_{5}\left(Z_{V V}\right)$ as obtained using the fit in eq.(3) (eq.(4)), as well as $\bar{E}_{5}$ $\left(\bar{E}_{V}\right)$. The results reported in tables 1 and $⿴$ ) have been obtained by fitting the two-point functions in the interval $12 \leq t / a \leq 20$ Tables 1 and 2 show that for the momenta $(1,0,0)$ and $(1,1,0)$ the continuum energy-momentum relation is satisfied within large statistical fluctuations. The statistical error increases as one approaches to the chiral limit and the agreement appears to be better for the vector meson than for the pseudoscalar one. We believe that this is due to our limited statistics.

In order to extract the current matrix elements from the three-point functions several approaches may be followed. We have used two different methods denoted in the following by "analytic" and "ratio". To be specific, we only explain these methods in the case of $\left\langle K\left|J_{\mu}\right| D>\right.$. The three-point correlation function is given by:

$$
\begin{aligned}
C_{\mu}\left(t_{x}, t_{y}\right) & =\sum_{\vec{x}, \vec{y}}<0\left|T\left[P_{5}^{K}(\overrightarrow{0}, 0) J_{\mu}\left(\vec{x}, t_{x}\right) P_{5}^{\dagger D}\left(\vec{y}, t_{y}\right)\right]\right| 0>e^{i \vec{q} \cdot \vec{x}+i \vec{p}_{D} \cdot \vec{y}} \\
& \rightarrow \frac{\sqrt{Z_{5}^{K} Z_{5}^{D}}}{4 E_{5}^{K} E_{5}^{D}}<K\left(\vec{p}_{D}+\vec{q}\right)\left|J_{\mu}\right| D\left(\vec{p}_{D}\right)>e^{-E_{5}^{K}\left|t_{x}\right|-E_{5}^{D}\left|t_{y}-t_{x}\right|}
\end{aligned}
$$

at large time distances. In eq.(河), $P_{5}^{K}\left(P_{5}^{\dagger D}\right)$ is the pseudoscalar density used to 
annihilate (create) a $\mathrm{K}$ meson (D meson) and $E_{5}^{K, D}$ the corresponding energiest. The matrix element can be isolated from the ratio:

$$
R=\frac{C_{\mu}\left(t_{x}, t_{y}\right)}{G_{5}^{K}\left(t_{x}, \vec{q}+\vec{p}_{D}\right) G_{5}^{D}\left(t_{y}-t_{x}, \vec{p}_{D}\right)} \times \sqrt{Z_{5}^{K} Z_{5}^{D}} \rightarrow<K\left|J_{\mu}\right| D>
$$

where $G_{5}^{K, D}$ are the analog of $G_{5}$ defined in eq.(3).

The two methods to extract the matrix elements are as follows:

- "analytic": in the denominator of eq.(6), we use the analytic expression of $G_{5}^{K, D}$ given in eq.(3), with $Z_{5}$ and $\bar{E}_{5}$ (computed from the meson mass) taken from the fit to the two-point function at zero momentum.

- "ratio": we divide the three-point correlation function by the two-point correlation functions with appropriate momenta averaged over the same configurations. We then multiply the result by the factor $\sqrt{Z_{5}^{K} Z_{5}^{D}}$ where $Z_{5}^{K}$ and $Z_{5}^{D}$ are obtained from the fit at zero momentum to the two-point functions corresponding to the $\mathrm{D}$ and $\mathrm{K}$ propagators.

With large time distances and a high statistics the two methods should agree, up to $O(a)$ effects. Our limited statistics gives fluctuations in the energy-momentum relations, in addition to these $O(a)$ effects. Therefore the two methods may yield slighlty different results and we will take into account the differences in the evaluation of the final error, see below. We report in tables 3 and the form factors for our set of light and heavy quark masses, from the analytic method and using the conserved vector current.

We are interested in the form factors at different $q^{2}$, for quark masses corresponding to the physical $D$ and $K(\pi)$ mesons. Thus we have to extrapolate the form factors reported in tables 3 and 4 , both in mass and momentum. We have proceeded as follows:

i) At fixed heavy quark mass and light meson momentum, $\vec{p}_{K, K^{*}}$, the generic form factor $F\left(F=f^{+}, A_{1}, \ldots\right)$ has been extrapolated linearly in the light quark mass to values corresponding to the strange $\left(D \rightarrow K, K^{*}\right)$ or massless $(D \rightarrow \pi, \rho)$ quarks. In ref. 四, it was shown that $S U(3)$ symmetry breaking effects are very small, i.e. that a linear dependence of the type:

$$
F=\alpha+\beta\left(\frac{1}{K_{1}}+\frac{1}{K_{2}}\right)
$$

describes well the behaviour of $F$ as a function of the light quark mass $\left(m_{q}^{1,2}=\right.$ $\left.1 / 2 a \times\left(1 / K_{1,2}-1 / K_{c r}\right)\right)$. In eq.(可) $K_{1}$ and $K_{2}$ correspond to the masses of the final and spectator quarks respectively. Thus the form factors extrapolated to the strange and light quark masses, $F^{K}$ and $F^{\pi}$, read $F^{K}=\alpha+\beta\left(1 / K_{s}+1 / K_{c r}\right)$

\footnotetext{
${ }^{4}$ We recall that all our calculations have been done with $\vec{p}_{D}=0$.
} 


\begin{tabular}{|c|c|c|c|c|}
\hline$K_{1}, K_{2}$ & $\vec{p}$ & $q^{2} a^{2}$ & $f^{+}\left(q^{2}\right)$ & $f^{0}\left(q^{2}\right)$ \\
\hline 0.1275 & $0,0,0$ & $0.296(4)$ & - & $0.91(8)$ \\
0.1485 & $1,0,0$ & $0.123(3)$ & $0.81(8)$ & $0.74(7)$ \\
& $1,1,0$ & $-0.010(2)$ & $0.76(17)$ & $0.77(17)$ \\
\hline 0.1275 & $0,0,0$ & $0.325(5)$ & - & $0.90(9)$ \\
0.1490 & $1,0,0$ & $0.137(3)$ & $0.82(11)$ & $0.73(9)$ \\
& $1,1,0$ & $-0.003(3)$ & $0.82(21)$ & $0.83(21)$ \\
\hline 0.1275 & $0,0,0$ & $0.361(7)$ & - & $0.91(11)$ \\
0.1495 & $1,0,0$ & $0.151(4)$ & $0.82(17)$ & $0.71(13)$ \\
& $1,1,0$ & $0.005(3)$ & $0.97(26)$ & $0.97(26)$ \\
\hline 0.1325 & $0,0,0$ & $0.186(3)$ & - & $0.93(8)$ \\
0.1485 & $1,0,0$ & $0.037(2)$ & $0.79(7)$ & $0.76(7)$ \\
& $1,1,0$ & $-0.078(1)$ & $0.74(17)$ & $0.80(16)$ \\
\hline 0.1325 & $0,0,0$ & $0.208(4)$ & - & $0.92(10)$ \\
0.1490 & $1,0,0$ & $0.046(2)$ & $0.78(10)$ & $0.74(9)$ \\
& $1,1,0$ & $-0.074(2)$ & $0.79(21)$ & $0.87(21)$ \\
\hline 0.1325 & $0,0,0$ & $0.236(6)$ & - & $0.93(11)$ \\
0.1495 & $1,0,0$ & $0.056(3)$ & $0.78(15)$ & $0.73(13)$ \\
& $1,1,0$ & $-0.069(2)$ & $0.94(26)$ & $1.03(28)$ \\
\hline 0.1375 & $0,0,0$ & $0.097(2)$ & - & $0.95(9)$ \\
0.1485 & $1,0,0$ & $-0.027(1)$ & $0.75(7)$ & $0.78(7)$ \\
& $1,1,0$ & $-0.122(1)$ & $0.70(17)$ & $0.83(16)$ \\
\hline 0.1375 & $0,0,0$ & $0.112(3)$ & - & $0.95(10)$ \\
0.1490 & $1,0,0$ & $-0.021(1)$ & $0.73(9)$ & $0.76(9)$ \\
& $1,1,0$ & $-0.120(1)$ & $0.75(21)$ & $0.92(22)$ \\
\hline 0.1375 & $0,0,0$ & $0.132(4)$ & - & $0.95(11)$ \\
0.1495 & $1,0,0$ & $-0.015(2)$ & $0.72(13)$ & $0.74(14)$ \\
& $1,1,0$ & $-0.118(1)$ & $0.89(26)$ & $1.10(31)$ \\
\hline 0.1425 & $0,0,0$ & $0.033(1)$ & - & $0.97(9)$ \\
0.1485 & $1,0,0$ & $-0.063(-)$ & $0.69(7)$ & $0.79(8)$ \\
& $1,1,0$ & $-0.137(-)$ & $0.65(17)$ & $0.88(17)$ \\
\hline 0.1425 & $0,0,0$ & $0.042(1)$ & - & $0.96(10)$ \\
0.1490 & $1,0,0$ & $-0.061(-)$ & $0.66(8)$ & $0.77(10)$ \\
& $1,1,0$ & $-0.137(-)$ & $0.68(21)$ & $0.98(25)$ \\
\hline 0.1425 & $0,0,0$ & $0.053(2)$ & - & $0.96(11)$ \\
0.1495 & $1,0,0$ & $-0.059(1)$ & $0.62(11)$ & $0.75(15)$ \\
& $1,1,0$ & $-0.137(-)$ & $0.80(27)$ & $1.16(40)$ \\
\hline & & & & \\
& & & & \\
& &
\end{tabular}

Table 3: Pseudoscalar $\rightarrow$ pseudoscalar form factors for different momenta and quark masses using the "analytic" method. The values reported in the table have been obtained using the "conserved" current. Errors denoted by (-) are smaller than the figures reported in the table. We also give the squared momentum transfer in lattice units. 


\begin{tabular}{|c|c|c|c|c|c|c|}
\hline$K_{1}, K_{2}$ & $\vec{p}$ & $q^{2} a^{2}$ & $V\left(q^{2}\right)$ & $\overline{A_{1}\left(q^{2}\right)}$ & $A_{2}\left(q^{2}\right)$ & $A\left(q^{2}\right)$ \\
\hline 0.1275 , & 0,0 & $0.237(6)$ & 2 & $0.61(6)$ & & \\
\hline \multirow[t]{2}{*}{0.1485} & $1,0,0$ & $0.088(4)$ & $0.80(7)$ & $0.52(4)$ & $0.43(12)$ & $0.09(3)$ \\
\hline & $1,1,0$ & $-0.035(3)$ & $0.61(15)$ & $0.47(15)$ & $0.36(34)$ & $-0.04(3)$ \\
\hline \multirow{3}{*}{$\begin{array}{c}0.1275 \\
0.1490\end{array}$} & $, 0,0$ & $0.254(8)$ & - & $0.59(6)$ & - & - \\
\hline & $1,0,0$ & 0.09 & $0.77(10)$ & 5) & 6) & 0.12 \\
\hline & $, 1,0$ & $-0.030(3)$ & $0.59(18)$ & $0.44(20)$ & $30(42)$ & -0.03 \\
\hline \multirow{3}{*}{$\begin{array}{l}0.1275, \\
0.1495\end{array}$} & $\overline{0,0}$ & 0.27 & - & $0.56(7)$ & - & - \\
\hline & 0,0 & 0.10 & $0.76(16)$ & $0.52(8)$ & $.51(26)$ & $0.17(8)$ \\
\hline & 0 & -0.02 & $56(24)$ & $0.37(28)$ & $0.19(57)$ & -0.031 \\
\hline \multirow{3}{*}{$\begin{array}{l}0.1325, \\
0.1485\end{array}$} & $\overline{0,0}$ & 0.1 & - & $0.66(6)$ & 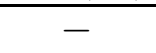 & - \\
\hline & 0,0 & 0.01 & $0.82(7)$ & $0.56(5)$ & $0.43(12)$ & $0.013(7)$ \\
\hline & $1,1,0$ & $-0.095(2)$ & $0.62(16)$ & $0.50(16)$ & $0.34(31)$ & $-0.12(9)$ \\
\hline \multirow{3}{*}{$\begin{array}{c}0.1325, \\
0.1490\end{array}$} & $\overline{0,0}$ & $0.152(5)$ & - & $0.64(7)$ & - & \\
\hline & $, 0,0$ & $0.016(3)$ & $0.79(10)$ & $0.55(6)$ & $0.44(16)$ & $0.02(1)$ \\
\hline & $1,1,0$ & $-0.092(2)$ & $.58(19)$ & $0.47(21)$ & $0.29(39)$ & $-0.12(12)$ \\
\hline \multirow{3}{*}{$\begin{array}{c}0.1325, \\
0.1495\end{array}$} & 0 & $\begin{array}{l}0.165(7) \\
\end{array}$ & 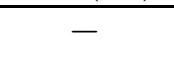 & $0.60(8)$ & - & \\
\hline & $1,0,0$ & 0.02 & $0.78(17)$ & $0.56(9)$ & $48(25)$ & $0.04(2)$ \\
\hline & $1,1,0$ & $-0.0 \varepsilon$ & $55(24)$ & $0.39(31)$ & $16(53)$ & $-0.11(16)$ \\
\hline \multirow{3}{*}{$\begin{array}{l}0.1375 \\
0.1485\end{array}$} & 0 & & 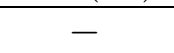 & & - & \\
\hline & 0 & -0.0 & $0.84(8)$ & 0.5 & $43(10)$ & $-0.06(2)$ \\
\hline & 0 & -0.1 & $.62(17)$ & 0.5 & $32(27)$ & $0.19(14)$ \\
\hline \multirow{3}{*}{$\begin{array}{c}0.1375, \\
0.1490\end{array}$} & $\overline{0,0,0}$ & & - & 7) & - & - \\
\hline & $1,0,0$ & -0.0 & $81(11)$ & 0.58 & $43(15)$ & $-0.07(3)$ \\
\hline & $1,1,0$ & -0.1 & $57(20)$ & $0.50(22)$ & $0.26(35)$ & $-0.19(19)$ \\
\hline \multirow{3}{*}{$\begin{array}{l}0.1375, \\
0.1495\end{array}$} & 0 & 0.0 & - & $0.64(8)$ & - & 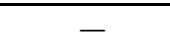 \\
\hline & 0 & -0.0 & $79(17)$ & & $13(23)$ & $-0.08(4)$ \\
\hline & $1,1,0$ & $-0.128(1)$ & $51(24)$ & $0.41(32)$ & $0.10(48)$ & $-0.18(27)$ \\
\hline \multirow{3}{*}{$\begin{array}{c}0.1425, \\
0.1485\end{array}$} & $\overline{0,0,0}$ & $0.015(1)$ & - & $0.75(8)$ & 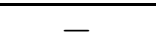 & 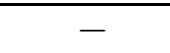 \\
\hline & $1,0,0$ & $-0.068(2)$ & $87(9)$ & $0.62(6)$ & $0.41(8)$ & $-0.13(5)$ \\
\hline & $1,1,0$ & $-0.135(-)$ & $0.61(18)$ & $0.56(17)$ & $0.27(22)$ & $-0.26(18)$ \\
\hline \multirow{3}{*}{$\begin{array}{c}0.1425, \\
0.1490\end{array}$} & $\overline{0,0,0}$ & $0.019(2)$ & - & $0.72(8)$ & - & - \\
\hline & $1,0,0$ & $-0.067(-)$ & 31(12) & $0.61(8)$ & $9(12)$ & $-0.15(6)$ \\
\hline & $1,1,0$ & $-0.136(-)$ & $0.53(21)$ & $0.52(22)$ & $0.20(28)$ & $-0.26(24)$ \\
\hline \multirow{3}{*}{$\begin{array}{c}0.1425, \\
0.1495\end{array}$} & $0,0,0$ & $0.023(2)$ & 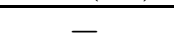 & & & - \\
\hline & & $-0.066(1)$ & 011 & 0.60 & & \\
\hline & 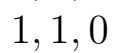 & $-0.136(-)$ & $0.43(2$ & 0.40 & ו & $0.21(3$ \\
\hline
\end{tabular}

Table 4: Pseudoscalar $\rightarrow$ vector form factors for different momenta and quark masses using the "analytic" method. The values reported in the table have been obtained using the "conserved" current. We also give the squared momentum transfer in lattice units. 


\begin{tabular}{|c|c|c|c|c|c|}
\hline$K_{1}$ & $K_{2}$ & $a M_{5}$ & $a M_{V}$ & $a M_{A}$ & $a M_{S}$ \\
\hline 0.1275 & 0.1485 & $0.813(4)$ & $0.822(4)$ & $0.94(2)$ & $0.91(2)$ \\
0.1275 & 0.1490 & $0.804(4)$ & $0.812(4)$ & $0.93(3)$ & $0.90(3)$ \\
0.1275 & 0.1495 & $0.794(5)$ & $0.803(5)$ & $0.92(3)$ & $0.90(4)$ \\
0.1275 & $K_{s}$ & $0.795(6)$ & $0.803(6)$ & $0.92(3)$ & $0.90(4)$ \\
0.1275 & $K_{c r}$ & $0.773(6)$ & $0.783(5)$ & $0.91(5)$ & $0.88(6)$ \\
\hline 0.1325 & 0.1485 & $0.700(4)$ & $0.712(4)$ & $0.83(2)$ & $0.81(3)$ \\
0.1325 & 0.1490 & $0.689(4)$ & $0.702(4)$ & $0.83(3)$ & $0.80(3)$ \\
0.1325 & 0.1495 & $0.679(5)$ & $0.693(5)$ & $0.82(4)$ & $0.79(5)$ \\
0.1325 & $K_{s}$ & $0.680(6)$ & $0.693(6)$ & $0.82(4)$ & $0.79(5)$ \\
0.1325 & $K_{c r}$ & $0.657(6)$ & $0.672(6)$ & $0.81(5)$ & $0.77(7)$ \\
\hline 0.1375 & 0.1485 & $0.580(4)$ & $0.598(5)$ & $0.73(3)$ & $0.69(3)$ \\
0.1375 & 0.1490 & $0.569(4)$ & $0.588(5)$ & $0.72(3)$ & $0.68(4)$ \\
0.1375 & 0.1495 & $0.558(5)$ & $0.578(5)$ & $0.72(4)$ & $0.67(6)$ \\
0.1375 & $K_{s}$ & $0.558(6)$ & $0.578(7)$ & $0.72(4)$ & $0.67(5)$ \\
0.1375 & $K_{c r}$ & $0.533(6)$ & $0.556(6)$ & $0.71(6)$ & $0.65(8)$ \\
\hline 0.1425 & 0.1485 & $0.450(4)$ & $0.479(5)$ & $0.63(3)$ & $0.56(4)$ \\
0.1425 & 0.1490 & $0.438(4)$ & $0.468(6)$ & $0.62(4)$ & $0.55(5)$ \\
0.1425 & 0.1495 & $0.425(5)$ & $0.457(6)$ & $0.62(5)$ & $0.54(7)$ \\
0.1425 & $K_{s}$ & $0.425(6)$ & $0.457(8)$ & $0.62(5)$ & $0.54(7)$ \\
0.1425 & $K_{c r}$ & $0.396(5)$ & $0.433(7)$ & $0.60(7)$ & $0.51(10)$ \\
\hline
\end{tabular}

Table 5: Pseudoscalar $\left(M_{5}\right)$, vector $\left(M_{V}\right)$, axial $\left(M_{A}\right)$ and scalar $\left(M_{S}\right)$ meson masses for mesons composed of heavy-light quarks (dimensionless units). 
and $F^{\pi}=\alpha+2 \beta / K_{c r}$, where $K_{s}=0.1495(1)$ is the value of the Wilson parameter for the strange quark and $K_{c r}=0.1506(1)$ the critical one. The value of $K_{c h a r m}$ corresponding to the charmed quark is $0.1379(11)$ f.

ii) Next we have extrapolated $F^{K, \pi}$ in the mass of the heavy quark according to the expression:

$$
F^{K, \pi}=A+\frac{B}{M_{P}}
$$

where $M_{P}$ is the mass of the heavy meson. At each value of $\vec{p}_{K, K^{*}}$, we have also extrapolated in $1 / M_{P}$ to the $\mathrm{D}$ and $\mathrm{B}$ mesons using the dependence expected in the heavy quark effective theory (HQET) [17], see eq.(11) below. The difference of the results obtained with the two methods are discussed later on. For $D$ decays, we have verified that extrapolating in a different order, e.g. first in $q^{2}$, then in the light quark mass, and finally in the heavy quark mass, leads to very similar results, within the statistical errors.

iii) To obtain the form factors at $q^{2}=0$, we have extrapolated the form factors at $\vec{p}_{K, K^{*}}=(1,0,0)$ (which in many cases corresponds to the smallest $q^{2}$, cf. tables 3 and (1) by assuming meson dominance:

$$
F\left(q^{2}\right)=\frac{F(0)}{1-q^{2} / M_{t}^{2}},
$$

where $F$ can be $f^{+}, V, A_{1}$ etc. and $M_{t}$ is the mass of the lightest meson exchanged in the t-channel. Thus the vector, scalar and axial scalar meson masses have been used for the extrapolation of $f^{+}$and $V, f^{0}$ and $A_{1,2}$ respectively. $M_{t}$ is computed on the lattice, over the same configurations, at the same heavy and light quark masses used for the three-point functions. In table 5 we report, in lattice units, the heavy-light pseudoscalar, vector, scalar and axial masses used in the present analysis, at different values of the Wilson parameters. In table 6 we also report the physical masses as extrapolated from the values given in table 5. To the results in table 6 we add $M_{D_{s}}-M_{D_{d}}=(93 \pm 6) \mathrm{MeV}$ and $M_{B_{s}}-M_{B_{d}}=(75 \pm 12)$ $\mathrm{MeV}$. In doing the extrapolation from small $q^{2}$ 's to $q^{2}=0$, the precise value of $M_{t}$ is relatively unimportant. For example we have verified that by using in all cases the vector meson mass, the results change by about $5 \%$.

In order to show the $q^{2}$ dependence of the different form factors and to compare it with the meson dominance hypothesis, we display in figs.1 the various form

\footnotetext{
${ }^{5}$ The errors given here are slighty different from those on ref. [8] where the time interval 14-24 was used for the fits of the two-point functions. In the present study we find $a^{-1}=3.6(2) \mathrm{GeV}$ instead of 3.7(2) as in ref. [8]. For the heavy-light mesons the interval $14-24$ is probably more appropriate, but we have checked that the difference for the form factors is immaterial given the statistical errors of the results.

${ }^{6}$ In ref. [8] were we used a time interval more apropriate to spectroscopy, we found $M_{D_{s}}-$ $M_{D_{d}}=(86 \pm 5) \mathrm{MeV}$ and $M_{B_{s}}-M_{B_{d}}=(62 \pm 7) \mathrm{MeV}$. Using a improved action 18 in the static limit it has been found $M_{B_{s}}-M_{B_{d}}=(76 \pm 10) \mathrm{MeV}$. These values can be compared to the experimental results $M_{D_{s}}-M_{D_{d}}=(99.9 \pm 0.7) \mathrm{MeV}\left[19\right.$ and $M_{B_{s}}-M_{B_{d}} \sim(96.3 \pm 4.8)$ $\mathrm{MeV}$ obtained from $M_{B_{s}}=5374.9 \pm 4.4 \mathrm{MeV} 20$ and $M_{B_{d}}=5278.6 \pm 2.0 \mathrm{MeV}[19$.
} 


\begin{tabular}{|c|c|c|c|c|l|}
\hline$M_{D^{*}}$ & $M_{D^{* *}} 1^{++}$ & $M_{D^{* *}} 0^{++}$ & $M_{D_{s}}{ }^{*}$ & $M_{D_{s} * *} 1^{++}$ & $M_{D_{s}{ }^{* *}} 0^{++}$ \\
\hline $1.95 \pm 0.01$ & $2.50 \pm 0.22$ & $2.30 \pm 0.28$ & $2.04 \pm 0.02$ & $2.55 \pm 0.16$ & $2.38 \pm 0.19$ \\
\hline \hline$M_{B^{*}}$ & $M_{B^{* *}} 1^{++}$ & $M_{B^{* *}} 0^{++}$ & $M_{B_{s}}{ }^{+}$ & $M_{B_{s} * *} 1^{++}$ & $M_{B_{s} * *} 0^{++}$ \\
\hline $5.27 \pm 0.01$ & $5.66 \pm 0.15$ & $5.61 \pm 0.17$ & $5.31 \pm 0.02$ & $5.72 \pm 0.10$ & $5.68 \pm 0.11$ \\
\hline
\end{tabular}

Table 6: Masses in GeV predicted from the lattice for the vector, axial and scalar excitations of the $D$ and $B$ mesons (remember that the pseudoscalar masses $M_{D}$ and $M_{B}$ are used as an input). These masses have been used in several occasions, as mentioned in the text, to extrapolate the form factors at $q^{2}=0$.

factors. They are shown as a function of the dimensionless variable $q^{2} / M_{t}^{2}$, for $K_{H}=0.1375$ and $K_{L}=0.1495$, which corresponds to the meson masses closest to the physical ones for $D \rightarrow K, K^{*}$ decays. For any given form factor, $M_{t}$ is the lattice meson mass appropriate for that particular channel. In the figures we have also included the points corresponding to a final meson with momenta $(1,1,0)$ and $(2,0,0)$. These points are reported for completeness but, given the extremely large systematic effects, they have never been used in the analysis. These figures show that the behaviour is compatible with meson dominance, at least in the range of masses and $q^{2}$ explored in our simulation. It is unknown whether the meson dominance will remain valid when we extrapolate to $\mathrm{B}$ decays, since the range of $q^{2}$ extends much further away from the pole in that case. Notice that in ref. 21, using QCD sum rules, it was found that the axial form factors do not follow the behaviour expected on the basis of the meson dominance.

\subsection{Main Systematic Effects}

Before we proceed to the physics of B and D meson decays let us summarize and discuss the main sources of systematic effects, besides "quenching", present in our calculation:

a) renormalization constants: the perturbative calculation is not unique in the sense that one has freedom in choosing the appropriate expansion parameter. It has been suggested that the bare coupling constant $g_{0}^{2}=6 / \beta$ is not very suitable and that it should be replaced by an effective coupling $g_{\text {eff }}^{2}$ [22]-24]. In our case, using the recipe of ref. [23, 24], $g_{\text {eff }}^{2} / g_{0}^{2}$ turns out to be $\sim 1.59$, which leads, using the formulae of refs. [14]- [16], to $Z_{V}=0.75$ and $Z_{A}=0.84$. The reliability of these estimates can be tested by the non-perturbative ratios (see also ref. [25, 26]):

$$
Z_{V}=\frac{<\alpha\left|V_{\mu}^{C}\right| \beta>}{<\alpha\left|V_{\mu}^{L}\right| \beta>}
$$

where $V^{L, C}$ are the local and conserved currents respectively. In our simulation, we found values of $Z_{V}$ which vary between 0.66 and 0.95 depending on the matrix element, see table 17. This range of values reflects uncertainties which have been 
Figure 1: We show an example of the $q^{2}$ behaviour of the form factors. We have chosen for the light and heavy Wilson parameters $K=0.1495$ and 0.1375 respectively. In each plot the two points furthest to the left (corresponding to the momentum assignment (1,1,1) and (2,0,0)) are only shown for the sake of illustration. We have never used them in the fits due to their large systematic and statistical errors. The curves correspond to the nearest pole dominance approximation with the mass $M_{t}$ of the relevant meson taken from table 5 and the numerator taken to fit the point closest to $q^{2}=0$ i.e. the momentum assignment $(1,0,0)$.

interpreted as $O(a)$ effects 27 and shown to be much smaller with the use of an "improved" action à la Symanzik 26]. In all our estimates we have used the perturbative values, $Z_{V}=0.84$ and $Z_{A}=0.88$. With the present systematic uncertainties this choice is appropriate. The reader can easily replace our numbers by those corresponding to his preferred value for $g_{\text {eff }}^{2}$.

b) $O(a)$ effects: from the above discussion we expect a systematic error of the order of $10-20 \%$ coming from $O(a)$ effects in the current matrix elements. The Fermilab group has suggested that this systematic error may be reduced by multiplying the propagators of the heavy quark $Q$ by $\exp \left(m_{Q} a\right)$ and redefining its mass [28]. This prescription, which does not change the results for $m_{Q} a \ll 1$, is motivated by the tree-level behaviour of lattice propagators. It is expected to work for small momenta, $p_{\mu} a \ll 1$, when the quark mass is large, $m_{Q} a \sim 1$. The difference between the results obtained using the standard procedure (i.e. multi- 


\begin{tabular}{|c|c|c|c|c|}
\hline$\vec{p}$ & $\left.<V_{1} V_{1}\right\rangle$ & $\left.<K J_{0} D\right\rangle$ & $\left\langle K J_{1} D\right\rangle$ & $\left\langle K_{3}^{*} J_{1} D\right\rangle$ \\
\hline$(0,0,0)$ & $0.659(3)$ & $0.87(-)$ & - & - \\
\hline$(1,0,0)$ & $0.652(3)$ & $0.94(1)$ & $0.75(1)$ & $0.66(2)$ \\
\hline$(1,1,0)$ & $0.645(5)$ & $0.95(3)$ & $0.79(3)$ & $0.69(5)$ \\
\hline
\end{tabular}

Table 7: Values of $Z_{V}$ calculated by taking the ratio of three-point correlation functions with inserted the "conserved" weak vector current divided by the corresponding correlation function with the local current inserted, cf. eq.(10). The Wilson parameters used for the three-point functions are $K_{H}=0.1375$ and $K_{L}=0.1495$. The results are shown for several momenta of the $K, K^{*}$, and for different Lorentz components of the weak current, $J_{i}$, and $K^{*}$ source, $K_{i}^{*}$. We also give $Z_{V}$ as derived from the light-light two-point function in the column labelled as $\left\langle V_{1} V_{1}\right\rangle$, computed with $K_{L}=0.1495$.

plying the lattice quark propagators only by $2 K$ ), and those obtained following the modified prescription at least represent some measure of the uncertainty due to discretisation errors.

We observe that the modification proposed by the Fermilab group leads to a universal change of all the matrix elements of a given current. Consequently, it cannot repair the fact that one finds very different values of $Z_{V}$ for different matrix elements as shown in table 7. Furthermore we have checked that $Z_{V}$ from $<V_{1} V_{1}>$ is almost independent of the quark mass. Indeed $Z_{V}$ from $<V_{1} V_{1}>$ for heavy-light mesons ranges from $0.643(2)$ to $0.653(3)$, which is almost identical to the light-light case reported in table 7. We conclude that in this case the dependence of $Z_{V}$ on the quark mass does not follow the behaviour predicted in ref. 28] 1. For this reason we have not followed the suggestion of ref. 28. We have instead decided to report here the form factors obtained from the local and "conserved" vector current and the local axial current. At the end, we will average the results obtained from the local and conserved vector current and add (in quadrature) as a systematic error $(\sim 10-20 \%$ for $V(0))$ their difference. In the case of the axial current we only have results from the local axial current, for which we also expect a 10-20\% systematic uncertainty coming from $O(a)$ effects.

c) extrapolation to the physical quark masses: the extrapolation in the light quark mass is quite natural and unlikely to be a source of an important uncertainty within our statistical accuracy. More delicate can be the extrapolation in the heavy quark mass. There are arguments, based on HQET which allow the expansion of the form factors at fixed $\vec{p}_{K, K^{*}}$, with $\left|\vec{p}_{K, K^{*}}\right| \ll M_{P}$, in inverse powers of the heavy meson mass $M_{P}[17]$. All the relevant formulae are given in sec.3.2, where we discuss the extrapolation to the B meson. Here we simply state

\footnotetext{
${ }^{7}$ This indicates that the vacuum to vector matrix element has a dependence on the quark mass different from that observed for the forward matrix element of the local current 28 . Similarly one may worry about the use of the recipe of ref. 28] for the pseudoscalar meson decay constants.
} 


\begin{tabular}{|c|c|c|c|c|}
\hline & $f_{K}^{+}(0)$ & $V(0)$ & $A_{1}(0)$ & $A_{2}(0)$ \\
\hline "a"-analytic & $0.73(72) \pm 0.16(16)$ & $0.85(1.10) \pm 0.24(30)$ & $0.63 \pm 0.14$ & $0.46 \pm 0.33$ \\
\hline "b"-analytic & $0.74(72) \pm 0.16(16)$ & $0.85(1.10) \pm 0.24(30)$ & $0.63 \pm 0.14$ & $0.46 \pm 0.33$ \\
\hline "a"-ratio & $0.60(59) \pm 0.12(12)$ & $0.84(1.09) \pm 0.24(30)$ & $0.63 \pm 0.14$ & $0.44 \pm 0.27$ \\
\hline "b"-ratio & $0.61(60) \pm 0.12(12)$ & $0.85(1.10) \pm 0.23(29)$ & $0.63 \pm 0.14$ & $0.44 \pm 0.27$ \\
\hline
\end{tabular}

Table 8: Semi-leptonic form factors at zero momentum transfer for $D \rightarrow K$ and $K^{*}$ using different extrapolations to the $D$ mesons: eq.(8), labelled as " $a$ ", and eq.(11), labelled as " $b$ ". The results are reported using the method called "analytic" or "ratio" to extract the form factors. The number obtained from the local vector current are reported in parenthesis.

that HQET suggests that $f^{+}$scales, at fixed $\vec{p}_{K, K^{*}}$, as:

$$
f^{+}=M_{P}^{1 / 2} \gamma_{+} \times\left(1+\frac{\delta_{+}}{M_{P}}\right)
$$

and similarly for $f^{-}, V$ and $A_{2}$, while $A_{1}$ scales like $M_{P}^{-1 / 2}$. For D mesons, the results, obtained by extrapolating the values given in tables 3 and 1 by using eq.(8) (fit "a") or eq.(11) ( fit "b") are reported in table 8 8. Although we have found that the $\chi^{2}$ is slightly better if we fit the form factors according to eq.(11), we do not have sufficiently good data to distinguish between the two behaviours. For completeness we also give the results obtained by using the local vector current (in parenthesis). From this table one sees that for $\mathrm{D}$ mesons the difference between the extrapolation "a" and "b" is rather small, $\leq 3 \%$. There is more difference in the case of the vector current between the results obtained using the conserved or the local current, in particular in the case of $D \rightarrow K^{*}$. As mentioned before we include this difference in the final error. For the axial current we are not able to estimate the error due to the determination of $Z_{A}$, since we do not have the equivalent of the conserved vector current. Besides the statistical errors reported in the tables, we then expect for the axial form factors a further error of order $10-20 \%$.

\section{Physics Results}

\subsection{Meson Decays}

Given the discussion of the previous section, our best estimates for the form factors and partial widths are those reported in the abstract and in tables 9 and 10. In this table we report our results together with other calculations and experimental determinations of the form factors. With respect to other

\footnotetext{
${ }^{8}$ Below we will give the same kind of table also for the B meson.
} 


\begin{tabular}{|c|c|c|c|c|}
\hline Ref. & $f_{K}^{+}(0)$ & $V(0)$ & $A_{1}(0)$ & $A_{2}(0)$ \\
\hline this work & $0.65 \pm 0.18$ & $0.95 \pm 0.34$ & $0.63 \pm 0.14$ & $0.45 \pm 0.33$ \\
\hline$[2]-[4]$ & $0.63 \pm 0.08$ & $0.86 \pm 0.10$ & $0.53 \pm 0.03$ & $0.19 \pm 0.21$ \\
\hline$[5]-[6]$ & $0.90 \pm 0.08 \pm 0.21$ & $1.43 \pm 0.45 \pm 0.49$ & $0.83 \pm 0.14 \pm 0.28$ & $0.59 \pm 0.14 \pm 0.24$ \\
\hline$[30]$ & 0.76 & 1.23 & 0.88 & 1.15 \\
\hline$[31]$ & $0.76-0.82$ & 1.1 & 0.8 & 0.8 \\
\hline$[21]$ & $0.6_{-0.10}^{+0.15}$ & $1.1 \pm 0.25$ & $0.5 \pm 0.15$ & $0.6 \pm 0.15$ \\
\hline Exp. [33 & $0.70 \pm 0.08$ & $0.9 \pm 0.3 \pm 0.1$ & $0.46 \pm 0.05 \pm 0.05$ & $0.0 \pm 0.2 \pm 0.1$ \\
\hline \hline Ref. & $A_{1}\left(q_{\max }^{2}\right)$ & $V(0) / A_{1}(0)$ & $A_{2}(0) / A_{1}(0)$ & $f^{0}\left(q_{\max }^{2}\right)$ \\
\hline this work & $0.62 \pm 0.09$ & $1.50 \pm 0.28$ & $0.7 \pm 0.4$ & $0.93 \pm 0.13$ \\
\hline$[4]$ & $0.77 \pm 0.20$ & $1.6 \pm 0.2$ & $0.4 \pm 0.4$ & - \\
\hline$[6]$ & $1.27 \pm 0.16 \pm 0.31$ & $1.99 \pm 0.22 \pm 0.33$ & $0.7 \pm 0.16 \pm 0.17$ & - \\
\hline$[30]$ & - & 1.1 & 1.3 & 1.15 \\
\hline [31] & - & 1.4 & 1.0 & - \\
\hline$[21]$ & - & $2.2 \pm 0.2$ & $1.2 \pm 0.2$ & - \\
\hline Exp. [33 & $0.54 \pm 0.06 \pm 0.06$ & - & - & - \\
\hline Exp. [34 & - & $2.00 \pm 0.33 \pm 0.16$ & $0.82 \pm 0.23 \pm 0.11$ & \\
\hline \hline
\end{tabular}

Table 9: Semi-leptonic form factors for $D \rightarrow K$ and $K^{*}$. For $f_{K}^{+}$and $V$ we have averaged the results obtained from the conserved and local currents and considered the difference as an error to add in quadrature to the statistical one.

calculations at a lower value of $\beta$, for example refs.[2]-[4] the errors are larger. This is expected because the statistics is lower and $\beta$ is higher. It is however reassuring that, at a value of $a^{-1}$ almost twice that of refs. [2]-[4], we find compatible results. Moreover experiments and lattice calculations are in good agreement for $f_{K}^{+}$, $V$ and $A_{1}$. On the other hand for $A_{2}$ the situation is unclear. E691 and ref. 田 suggest a smaller value of $A_{2}$ than E653, ref. [5] and the present work, even though the errors on this quantity are so large that all the results are compatible. Thus for $A_{2}$ it is very important to reduce both the experimental and theoretical errors. The agreement with the other form factors is already remarkable, given the fact that the lattice calculations have no free parameter.

\subsection{Extrapolation to B meson decays}

With the present lattice spacing we are unable to study directly the B mesons in numerical simulations. However indirect information on B physics may be available using the following strategy. One first studies the scaling behaviour of a given physical quantity in the region of the charm quark mass. Then one computes the same quantity in the effective ("static") theory, i.e. in the limit in which the heavy quark mass is infinite. By interpolating between the two results with the help of the expected scaling laws, one can predict that physical quantity 


\begin{tabular}{|c|c|c|c|}
\hline Ref. & $\Gamma(D \rightarrow K) / 10^{10} s^{-1}$ & $\Gamma\left(D \rightarrow K^{*}\right) / 10^{10} s^{-1}$ & $\Gamma(D \rightarrow \pi) / 10^{10} s^{-1}$ \\
\hline this work & $6.8 \pm 3.4$ & $6.0 \pm 2.2$ & $0.56 \pm 0.36$ \\
\hline [4] & $5.8 \pm 0.15$ & $5.0 \pm 0.9$ & $0.5 \pm 0.2$ \\
\hline exp. [35, 32 & $7.0 \pm 0.8$ & $4.0 \pm 0.7$ & $0.9_{-0.3}^{+0.5}$ \\
\hline \hline Ref. & $\Gamma(D \rightarrow \rho) / 10^{10} s^{-1}$ & $\Gamma\left(D \rightarrow K^{*}\right) / \Gamma(D \rightarrow K)$ & $\Gamma_{L} / \Gamma_{T}$ \\
\hline this work & $0.50 \pm 0.23$ & $0.92 \pm 0.55$ & $1.27 \pm 0.29$ \\
\hline [4] & $0.4 \pm 0.09$ & $0.86 \pm 0.22$ & $1.51 \pm 0.27$ \\
\hline [30 & - & 1.14 & 0.89 \\
\hline [31] & - & 1.45 & 1.11 \\
\hline [21] & - & $0.5 \pm 0.15$ & $0.86 \pm 0.06$ \\
\hline [36 & - & $0.63 \pm 0.09$ & - \\
\hline exp. [35 & - & $0.57 \pm 0.08$ & $1.15 \pm 0.17$ \\
\hline \hline
\end{tabular}

Table 10: Semi-leptonic partial widths for $D \rightarrow K, K^{*}, \pi$ and $\rho$, using $V_{c s}=$ 0.975 and $V_{c d}=0.222$. We also report the ratio of the longitudinal to transverse polarisation partial widths for $D \rightarrow K^{*}$.

for the $\mathrm{B}$ meson. The value in the static limit reduces the uncertainty due to the extrapolation from the charm region. This strategy has proved to be effective for the pseudoscalar decay constant, see for example refs. 88, 29.

At our large value of $\beta$, the small statistics is not sufficient to compute semileptonic form factors in the static limit. Nevertheless we can study the scaling behaviour of the form factors and try an extrapolation to the $b$ quark. With the actual errors, our predictions remain necessarily at a semi-quantitative level. The study is however interesting in itself. We have found that the expected dependence of the form factors in $1 / M_{P}$, see eq.(11), is compatible with our results. For some of the form factors however the corrections to the static results in the charm region may be sizeable. In the following we explain the extrapolation of the form factors, computed at several values of the heavy quark mass, to the B meson.

On the basis of HQET, up to $O\left(1 / M_{P}^{2}\right)$, up to logarithmic corrections, one expects the following behaviour for the relevant form factors [17]:

$$
\begin{array}{lll}
\frac{f^{+}}{M_{P}^{1 / 2}}=\gamma_{+} \times\left(1+\frac{\delta_{+}}{M_{P}}\right) & \frac{V}{M_{P}^{1 / 2}}=\gamma_{V} \times\left(1+\frac{\delta_{V}}{M_{P}}\right) \\
\frac{A_{2}}{M_{P}^{1 / 2}}=\gamma_{2} \times\left(1+\frac{\delta_{2}}{M_{P}}\right) & A_{1} M_{P}^{1 / 2}=\gamma_{1} \times\left(1+\frac{\delta_{1}}{M_{P}}\right)
\end{array}
$$

The expansions given in eqs.(12) become valid in the limit of large $m_{Q}$, at fixed momentum $\vec{p}$ of the light meson (in the frame where the heavy meson is at rest) and when $|\vec{p}| \ll m_{Q} \sim M_{P}$. The above conditions are always satisfied for $q_{\max }$, when the initial and final mesons are both at rest. Provided we are not too close to the meson pole in the t-channel, the dependence on the light mass is smooth 
Figure 2: Form factors extrapolated to the chiral limit for the light quark, as a function of the inverse pseudoscalar mass $\left(1 / M_{P}\right)$ for the momentum assignment $(1,0,0)$. The crosses are the lattice points, the diamonds are the extrapolation to the $D$ and $B$ meson. Notice that the $D$ is very close to a lattice point. The points corresponding to the lightest heavy quark mass (furthest to the right) has not been used in the fits.

and it is well described by eq.(7). We can then extrapolate in the heavy quark mass according to eqs.(12). The points corresponding to $\vec{p}=(1,0,0)$ also satisfy the above conditions. However, in the range of masses where we compute the form factors, $m_{Q} \sim m_{\text {charm }}$, these points correspond to $q^{2} \sim 0$. This is due to the fact that the heavy meson masses are not so heavy in comparison with $|\vec{p}|$ and that the light mesons are not really light enough. Thus, while the typical $\sqrt{q^{2}}$ on our lattice is at most $1.4 \mathrm{GeV}$, the extrapolation in the heavy quark mass will bring us to $\sqrt{q^{2}}=4.2 \mathrm{GeV}\left(q^{2}=M_{B}^{2}-2 * M_{B} \sqrt{M_{\pi}^{2}+(2 \pi / 24 a)^{2}}+M_{\pi}^{2}\right)$, and similarly for $B \rightarrow \rho$. The uncertainty involved in such an extreme extrapolation must be borne in mind. The validity of the extrapolation is partially justified only because the range in $1 / M_{P}$ is relatively small and most of the form factors have a smooth behaviour. The case $(1,1,0)$ is more complicated because, with the heavy quark masses at hand, we have $|\vec{p}| \sim M_{P}$ where the expansion of ref. [17] is questionable. Anyhow the errors are so large that we have not used these points.

In fig.2 we give the form factors, at the value of $q^{2}$ corresponding to the 


\begin{tabular}{|c|c|c|c|c|}
\hline$\vec{p}$ & $\gamma_{+} \mathrm{GeV}^{-1 / 2}$ & $\gamma_{V} \mathrm{GeV}^{-1 / 2}$ & $\gamma_{1} \mathrm{GeV}^{+1 / 2}$ & $\gamma_{2} \mathrm{GeV}^{-1 / 2}$ \\
\hline$(0,0,0)$ & - & - & $0.96 \pm 0.16$ & - \\
\hline$(1,0,0)$ & $0.39 \pm 0.25$ & $0.29 \pm 0.12$ & $1.05 \pm 0.25$ & $0.44 \pm 0.25$ \\
\hline \hline$\vec{p}$ & $\delta_{+} \mathrm{GeV}$ & $\delta_{V} \mathrm{GeV}$ & $\delta_{1} \mathrm{GeV}$ & $\delta_{2} \mathrm{GeV}$ \\
\hline$(0,0,0)$ & - & - & $-0.33 \pm 0.09$ & - \\
\hline$(1,0,0)$ & $0.0 \pm 1.1$ & $1.9 \pm 1.3$ & $-0.46 \pm 0.22$ & $-0.6 \pm 0.8$ \\
\hline
\end{tabular}

Table 11: The coefficients of the $1 / m_{Q}$ expansion of the form factors defined in eqs.(12).

momentum assignment $(1,0,0)$, extrapolated to the chiral limit in the light quark mass, as a function of $1 / M_{P}$ (crosses). In the figure we also give the value extrapolated to the $D$ and $B$ mesons (diamonds). The figure shows that the points are smoothly extrapolated, even in the case of $V$ since $\gamma_{V}$ is small. This is encouraging for further studies with higher statistics. In table 11 we report the values of $\gamma_{+, V, 1,2}$ and $\delta_{+, V, 1,2}$ for $\vec{p}=(0,0,0)$ and $(1,0,0)$, in physical units. We tried also $(1,1,0)$, but the errors turned out be of order $100 \%$ and we have not reported them in the table. We notice that the first correction in $1 / M_{P}$ is small in the case $A_{1}$. This is also the case for $f^{+}$, at $(1,0,0)$, even though, because of the large error, we cannot exclude a slope $\sim 0.7 \mathrm{GeV}$. The same can be said for $A_{2}$, whose value anyhow is badly determined even before the extrapolation. In the case of $V$ we find instead a rather large correction. We believe that this is an interesting and rather unexpected result, which deserves an effort to reduce the size of the statistical error. Taking our present errors into account we cannot exclude that the large value of $\delta_{V}$ is correlated to the small value of $\gamma_{V}$ and that it will disappear with more accurate results.

From the numbers given in the table we can predict the form factors of the B mesons. We give all the form factors at $q^{2}=0$. They have been obtained from the form factors at $\vec{p}=(1,0,0)$, by using the meson dominance, with the mass of the mesons exchanged in the t-channel reported in table 6. These masses have been obtained by fitting the mass difference $\Delta M=M_{P^{*}}-M_{P}$ (vector case) and $\Delta M=M_{P^{* *}}-M_{P}$ (axial case) as $\Delta M=A_{M}+B_{M} / M_{P}$. The results are reported in table 6. They are rather close to the experimental masses when known, and also to the masses computed in ref.[30], i.e. $M_{B^{*}}=5.32 \mathrm{GeV}$ and $M_{B^{* *}, 1^{++}}=5.71$ $\mathrm{GeV}$, except for the scalar mass which in ref. 30] is very large: $M_{B^{* *}, 0^{++}}=5.99$ $\mathrm{GeV}$. The differences between the extrapolations using different sets of masses turn out to be small and may be smaller than the error induced by the assumption of the meson dominance. The results are reported in table 12, label "b", together with the results of refs. 30]-32 for comparison. In the evaluation of the errors we have also taken into account of the difference between the results obtained using the local or the conserved current. To show the stability of the results with respect to a different extrapolation, we have also reported the values obtained 


\begin{tabular}{|c|c|c|c|c|}
\hline Ref. & $f^{+}(0)$ & $V(0)$ & $A_{1}(0)$ & $A_{2}(0)$ \\
\hline this work "a" & $0.28 \pm 0.14$ & $0.37 \pm 0.14$ & $0.24 \pm 0.06$ & $0.39 \pm 0.24$ \\
\hline this work "b" & $0.33 \pm 0.17$ & $0.40 \pm 0.16$ & $0.21 \pm 0.05$ & $0.47 \pm 0.28$ \\
\hline [30] & 0.33 & 0.33 & 0.28 & 0.28 \\
\hline [3] & 0.09 & 0.27 & 0.05 & 0.02 \\
\hline [32] & $0.26 \pm 0.02$ & $0.6 \pm 0.2$ & $0.5 \pm 0.1$ & $0.4 \pm 0.2$ \\
\hline \hline Ref. & - & $V(0) / A_{1}(0)$ & $A_{2}(0) / A_{1}(0)$ & - \\
\hline this work "a" & - & $1.4 \pm 0.5$ & $1.5 \pm 0.8$ & - \\
\hline this work "b" & - & $1.7 \pm 0.6$ & $2.3 \pm 1.1$ & - \\
\hline [30] & - & 1.0 & 1.0 & - \\
\hline \hline
\end{tabular}

Table 12: Semi-leptonic form factors for $B \rightarrow \pi$ and $\rho$. For $f^{+}$and $V$ we have averaged the results obtained from the conserved and local currents and considered the difference as an error to add in quadrature to the statistical one. The label "a" refers to the naive extrapolation in $1 / M_{P}$, eq.(8), and label " $b$ " to the extrapolation given in eqs.(12) respectively. To extrapolate to zero momentum transfer we have used the masses of table 6 .

with the naive scaling given in eq.(8), labelled as "a".

In the $\mathrm{D}$ meson the difference between the HQET scaling laws and naive scaling was immaterial. The differences remain small in the B case and with our errors we cannot distinguish the two behaviours. Notice that, because of the different scaling laws, $A_{2} / A_{1}$ increases with the mass of the heavy quark and can be $\sim 2$ for $B \rightarrow \rho$.

For most of the form factors the predictions of ref. 31] are much lower than all the others, cf. table 12. These result in a much larger estimate of $\left|V_{u b}\right|$, for a given experimental branching ratio. Notice however that in ref.[31] the form factor is computed at $q_{\text {max }}^{2}$ and then a "tempered" exponential dependence on $q^{2}$ is assumed. This $q^{2}$ yields a dramatic suppression at small $q^{2}$ for B meson decays, where the range in $q^{2}$ is very large.

From the numbers reported in table 12, we can give a very rough estimate of the $B \rightarrow \pi$ and $B \rightarrow \rho$ branching ratios. The branching ratios are obtained from the form factors in the table, by assuming meson dominance for their dependence on $q^{2}$, see eq.(9). To get an estimate of the errors, we have allowed the form factors to vary in all the possible ways by one $\sigma$ within the statistical errors and to vary in all possible ways among the values obtained with different extrapolations in $1 / M_{P}$, fits "a" and "b". In this way we can partially account for the uncertainty coming from the extrapolation and the $q^{2}$ dependence of the form factors. We finally get:

$$
\Gamma\left(B \rightarrow \pi l \nu_{l}\right)=\left|V_{u b}\right|^{2}(12 \pm 8) \times 10^{12} s^{-1}
$$

and

$$
\Gamma\left(B \rightarrow \rho l \nu_{l}\right)=\left|V_{u b}\right|^{2}(13 \pm 12) \times 10^{12} s^{-1}
$$


Thus the errors are still too large to give more than an upper bound. Since experiment, on its own side, only gives upper bounds for the considered branching ratios, we cannot with our present accuracy extract any information on $V_{u b}$. We mainly want to stress the feasibility of this analysis, provided one disposes of larger statistics.

\section{Acknowledgments}

We thank R. Sarno for her participation in the early stages of this work. We thank A. Le Yaouanc for fruitful discussions. C.R.A., M.C., V.L. and G.M. acknowledge the partial support of the MURST, Italy, and INFN. The work of S.G., G.S. and K.S was supported by Deutsche Forschungsgemeinschaft, grant Schi 257/3-1 3-2. C.T.S. acknowledges the support of the Science and Engineering Research Council through the arward of a Senior Fellowiship. We

warmly thank the staffs of CINECA, PSI (Orsay), CCVR (Polytechnique) and Höchstleistungsrechenzentrum Jülich for their continuous and effective support.

\section{References}

[1] see, e.g. G.Martinelli, Nucl.Phys. B26 (Proc.Suppl.) (1992)31;

C.T.Sachrajda, Nucl.Phys. B30 (Proc.Suppl.) (1993)20;

C.Bernard and A.Soni, in Quantum Fields on the Computer, M.Creutz (edt.), World Scientific, Singapore, 1992.

[2] M.Crisafulli et al., Phys.Lett. 223B (1989) 90.

[3] V.Lubicz, G.Martinelli and C.T.Sachrajda, Nucl.Phys. B356 (1991) 310.

[4] V.Lubicz, G.Martinelli, M.McCarthy and C.T.Sachrajda, Phys.Lett. 274B (1992) 415.

[5] C.Bernard, A.El-Khadra and A.Soni, Phys.Rev.D43 (1992) 2140.

[6] C.Bernard, A.El-Khadra and A.Soni, Phys.Rev. D45 (1992) 869.

[7] R. Gupta, Nucl.Phys.B(Proc.Suppl.) 30 (1993) 419.

[8] As. Abada et al., Nucl.Phys. B376 (1992) 172.

[9] K.G. Wilson, in "New Phenomena in Sub-nuclear Physics", ed. A. Zichichi, Plenum, New york (1977).

[10] M. Creutz, Phys.Rev D36 (1987) 515. 
[11] P. Gibbs, University of Glasgow Preprint (1985).

[12] L.H. Karsten and J. Smit, Nucl.Phys. B183 (1981) 103

[13] M. Bochicchio et al. Nucl.Phys. B262 (1985) 331.

[14] G. Martinelli and Y.C. Zhang, Phys.Lett. B123 (1983) 433.

[15] B. Meyer and C. Smith, Phys.Lett. 123 (1983) 62.

[16] R. Groot, J. Hoek and J. Smit, Nucl.Phys. B237 (1984) 111.

[17] N. Isgur and M.B. Wise, Phys.Rev. D42 (1990) 2388.

[18] C.R. Allton et al., LPTENS 93/12 - ROME prep. 93/928 (1993), submitted to Nucl. Phys. B.

[19] Review of Particle Properties, Phys.Rev. 45 (1992).

[20] See for example S.Stone, 5th International Symposium on Heavy Flavour Physics, Montreal, Canada, 6-10 July 1993, to appear in the proceedings.

[21] P. Ball, V.M. Braun, H.G. Dosch Phys.Rev. D44 (1991) 3567.

[22] G. Parisi, XX Int. Conf., Madison, Wisconsin, L. Durand and L.G. Pondrom eds., American Institute of Physics (1981).

[23] G.P. Lepage and P.B. Mackenzie, Nucl.Phys. B (Proc. Suppl) 20 (1991) 173.

[24] G.P. Lepage and P.B. Mackenzie, Fermilab Preprint, PUB-91/355-T (revised) (1992).

[25] L. Maiani and G. Martinelli, Phys.Lett. B178 (1986) 265.

[26] G. Martinelli, C.T. Sachrajda and A. Vladikas, Nucl.Phys. B358 (1991) 212.

[27] G. Heatlie et al., Nucl.Phys. B352 (1991) 266.

[28] A. Kronfeld, Nucl.Phys.B(Proc.Suppl.) 30 (1993) 444.

[29] C. Alexandrou, F. Jegerlehner, S. Güsken, K. Schilling, and R. Sommer: Bounds on $f_{B}$ from Lattice QCD, Nucl.Phys. B374(1992)263.

[30] M. Bauer, B. Stech and M. Wirbel, Z. Phys. C29 (1985) 637; C34 (1987) 103.

[31] N. Isgur, D. Scora, B. Grinstein and M.B. Wise, Phys.Rev. D39 (1989) 799; N. Isgur and D. Scora Phys.Rev. D40 (1989) 1491. 
[32] P. Ball, TUM-T31-39-93, Bulletin Bd.: hep-ph@xxx.lanl.gov - 9305267.

[33] J.C. Anjos et al. Phys.Rev.Lett. $\underline{65}$ (1990) 2630.

[34] K. Kodama et al. Phys.Lett. B274 (1992) 246.

[35] See for example S.Stone, in Heavy Flavour Physics, eds. A.J. Buras and H. Lindner, world scientific, Singapore (1992).

[36] CLEO II, as reported by P. Burchat, 5th International Symposium on Heavy Flavour Physics, Montreal, Canada, 6-10 July 1993, to appear in the proceedings. 